\title{
Short Note \\ The application and benefits of digital geological mapping in Antarctica
}

\author{
MICHAEL L. CURTIS, TEAL R. RILEY, MICHAEL J. FLOWERDEW and ALEX TATE \\ British Antarctic Survey, NERC, High Cross, Madingley Road, Cambridge CB3 OET, UK \\ m.curtis@bas.ac.uk
}

Received 2 November 2010, accepted 23 February 2011, first published online 5 April 2011

\section{Introduction}

Antarctica remains a remote and logistically difficult region in which to conduct geological fieldwork, making the data collected there of significant value. The British Antarctic Survey (BAS) maintains a geological database containing sample and locality data from over sixty years of fieldwork, although the vast majority of field observations and data remain in notebooks and paper maps. Little or none of these data or observations are easily searchable, thus their exploitation is time consuming and inefficient, reducing its corporate and research value. In order to record and store field data in an efficient and consistent manner, geologists at BAS developed a digital geological mapping system (DGMS) that integrates with BAS's existing database. Various versions of the system have been successfully deployed during several field campaigns in South Georgia, South Orkney Islands, the Antarctic Peninsula and Ellsworth Mountains. In this contribution we summarize our experiences in deploying digital geological mapping technology in Antarctic and sub-Antarctic environments.

\section{Equipment}

Geological fieldwork in Antarctica is invariably conducted from remote field camps sited in heavily glaciated and mountainous areas that impose practical restrictions on the type of equipment suitable for a DGMS. Antarctic field workers are generally encumbered with a substantial burden of mountaineering and survival equipment, therefore it is essential that DGMS equipment be lightweight and small in volume, while battery life must exceed a full day's usage. Clegg et al. (2006) discussed in detail the advantages and disadvantages of DGMSs based on tablet PCs or personal digital assistants (PDAs), and despite the subsequent advances in mobile computing technology their conclusions still hold true. Ruggedized tablet PCs, although best suited for digital geological mapping and regularly employed by some national surveys, remain too cumbersome to be carried by an Antarctic field worker for a full day, an issue compounded by the necessity for replacement batteries. Therefore BAS, like the Geological Survey of Denmark and Greenland who face similar practical restrictions (Schlatter et al. 2010), based our
DGMS around a PDA (e.g. Trimble Nomad or HP iPaq housed in a rugged OtterBox ${ }^{\circledR}$ case) running ESRI ${ }^{\circledR}$ ArcPad 7, linked wirelessly to a consumer Bluetooth GPS. The battery life of both devices comfortably exceeds a full day's use and can be recharged at base camp from an external battery pack powered by solar panels. While consumer grade PDAs worked well in the sub-Antarctic and northern Antarctic Peninsula, fully ruggedized models such as the Trimble Nomad are ideal and are best suited to the colder interior regions of Antarctica.

The BAS DGMS utilizes a custom designed user interface created using ESRI ${ }^{\circledR}$ ArcPad Application Builder to capture point, polyline and polygon data in ArcPad. The interface is based on modular forms containing context sensitive dropdown lists, populated from pre-defined look-up tables (LUTs), which minimize data entry effort and maximize the flexibility of the data entry forms.

A critical aspect of field geology is recording information that places data into its local and/or regional context. This is accomplished in the BAS DGMS by the provision of free text fields that may be specific to an individual data record or general contextual observations relating to an individual locality. The Windows Mobile operating system provides efficient software tools (i.e. transcriber or Soft Input Panel) for inputting limited amounts of text in the absence of a keyboard. In addition, simple digital sketches can be recorded within the DGMS via integration with the freeware drawing package PDApaint. Digital field photographs are logged within the DGMS enabling hyperlinking in ArcGIS, while they are also georeferenced using the freeware application GeoSetter using a GPS track log.

\section{Benefits of a DGMS}

The digital capture of geological data in the field has numerous benefits for individual workflow and corporate data value (Clegg et al. 2006, Athey et al. 2008, Schlatter et al. 2010). Our experience within BAS highlights several key benefits:

1) Accuracy both in geographical location and data recording are inherent within a DGMS. GPS integration enables all data to be geospatially referenced with latitude, longitude and elevation, removing the potential 
for error associated with manual data input. The system also quantifies GPS fix accuracy by recording the position dilution of precision (PDOP).

2) Data consistency is achieved by the provision of LUTs populated by standardized nomenclature, as well as providing a standard format for structural data. Ensuring the consistency of data, observations and data models between individual field geologists and across projects enables easy integration and analysis of datasets and is essential for efficient data management.

3) The design of data entry forms within DGMS encourages more systematic recording of geological observations, and potentially reduces sampling bias, e.g. the data used in an analysis of tectonic fold appression to determine strain paths (Curtis et al. 2010) is directly attributable to systematic data capture of fold attributes using a DGMS.

4) Although the quantity, quality and accuracy of regional topographic maps of Antarctica are improving, DGMSs are not reliant on base maps and enable detailed geological mapping in areas where none exist. However, where geographically referenced geological and geophysical maps are available they can be displayed as a base layer in ArcPad helping to focus field effort and aid interpretation.

5) All data and observations are automatically stored within a GIS enabling detailed spatial and attribute analyses in ESRI ${ }^{\circledR}$ ArcMap (e.g. Chattopadhyay et al. 2010).

6) Data security is enhanced due to ease of backup between removable solid-state memory cards within the PDA and/or by synchronising the PDA with a laptop or PC.

\section{Limitations of PDA based DGMSs}

Alongside the demonstrable benefits of using a DGMS, there are also real and perceived drawbacks that may limit the adoption of any digital system:

1) While our experience is that DGMS hardware and software are relatively robust, system reliability is a significant and legitimate concern when working in a remote environment where technical support is severely limited. However, the risk of technical failure can be mitigated by deploying spare hardware devices, which given the relatively low unit cost of our chosen hardware is financially practical, while adequate training in DGMS use and troubleshooting common issues is essential.

2) Ideally a DGMS should be able to capture all observations and data in a consistent and accessible format. However, it is inevitable due to the potential complexity of geological localities that some geological features and relationships will not be adequately recorded without the use of free text observations or interpretations. While the BAS DGMS offers free text facilities where locality or record specific contextual information may be entered, PDAs are not best suited for entering passages of text. For time efficiency, long free text notes are still recorded in a traditional notebook and digitally transcribed at camp when one has access to a laptop or keyboard.

3) Adopting a DGMS requires a significant change in geological mapping practice. Given that many individuals have developed their own field techniques for data acquisition over the course of their careers it is unlikely that all will embrace this change.

\section{Summary}

The digital capture of field data in Antarctic using a DGMS is both practicable and desirable, and its systematic use considerably increases the corporate and research value of the data captured. Dependent on the extent of automation, DGMSs based on PDA style mobile computers offer potential time efficiencies in the field and improve work flow from data capture, backup, archiving to spatial analysis, although as yet they are not a complete replacement for a traditional notebook.

\section{References}

Athey, J.E., Freeman, L.K. \& Woods, K.A. 2008. The transition from traditional to digital mapping: maintaining data quality while increasing geologic mapping efficiency in Alaska. Alaska Geosurvey News, 11, 2-8. Chattopadhyay, A., Holdsworth, R.E., McCaffrey, K.J.W. \& Wilson, R.W. 2010. Recording and analysing geospatially accurate structural data through 'Digital Mapping' technique: a case study from the Canisp Shear Zone, NW Scotland. Journal of Geological Society of India, 75, 43-59.

Clegg, P., Bruciatelli, L., Domingos, F., Jones, R.R., de Donatis, M. \& Wilson, R.W. 2006. Digital geological mapping with tablet PC and PDA: a comparison. Computers \& Geosciences, 32, 1682-1698.

Curtis, M.L., Flowerdew, M.J., Riley, T.R., Whitehouse, M.J. \& Daly, S. 2010. Andean sinsitral transpression and kinematic partitioning in South Georgia. Journal of Structural Geology, 32, 464-477.

Schlatter, D., Bo, U.L., Stensgaard, M. \& Buller, G. 2010. Digital field data capture: the Geological Survey of Denmark and Greenland experiences in Greenland. Explore, 147, 2-14. 